\title{
Mulheres de meia-idade e a calça jeans: uma revisão sistemática sobre a percepção
}

\section{de conforto/desconforto}

\author{
Middle-aged women and blue jeans: a systematic review on the perception of comfort/discomfort \\ Mujeres de mediana edad y jeans: una revisión sistemática sobre la percepción de
}

comodidad/malestar

Recebido: 13/10/2021 | Revisado: 20/10/2021 | Aceito: 25/10/2021 | Publicado: 28/10/2021

\author{
Onnara Custódio Gomes \\ ORCID: https://orcid.org/0000-0001-6881-3831 \\ Universidade Estadual Paulista "Júlio de Mesquita Filho", Brasil \\ E-mail: onnara.custodio@unesp.br \\ Marizilda dos Santos Menezes \\ ORCID: https://orcid.org/0000-0003-4242-0698 \\ Universidade Estadual Paulista "Júlio de Mesquita Filho", Brasil \\ E-mail: marizilda.menezes@unesp.br \\ Luis Carlos Paschoarelli \\ ORCID: https://orcid.org/0000-0002-4685-0508 \\ Universidade Estadual Paulista "Júlio de Mesquita Filho", Brasil \\ E-mail: luis.paschoarelli@unesp.br
}

\begin{abstract}
Resumo
Este artigo teve como objetivo identificar e analisar estudos sobre a percepção de conforto/desconforto, no uso da calça jeans, por mulheres de meia-idade, a partir de uma revisão bibliográfica sistemática (RBS). A busca por evidências foi realizada nas bases de dados Scopus, Web of Science e Periódicos Capes. Utilizou-se instrumento adaptado de RBS. Foram selecionados dez artigos que atenderam aos critérios de inclusão e de qualidade. Constatou-se que existem lacunas sobre pesquisas ligadas às mulheres de meia-idade, a relação desse público com o uso da calça jeans e sua percepção de conforto/desconforto, respondendo parcialmente à questão de pesquisa. Foram identificadas limitações e sugeridas mais pesquisas que contemplem a temática, contribuindo com pesquisas e desenvolvimento de produtos jeanswear e com a melhoria da qualidade de vida das usuárias.
\end{abstract}

Palavras-chave: Ergonomia; Agradabilidade; Envelhecimento; Jeanswear.

\begin{abstract}
This article aimed to identify and analyze studies on the perception of comfort/discomfort during the use of blue jeans by middle-aged women, based on a systematic literature review (SLR). The search for evidence was performed in the Scopus, Web of Science and Periódicos Capes databases. An adapted SLR instrument was used. Ten articles that met the inclusion and quality criteria were selected. It was found that there are gaps in research related to middle-aged women, the relationship of this public and the use of blue jeans and their perception of comfort/discomfort, partially answering the research question. Limitations were identified and more research was suggested that address the theme, contributing to researches and development of jeanswear products, and improving the quality of life of female users.
\end{abstract}

Keywords: Ergonomics; Pleasantness; Aging; Jeanswear.

\section{Resumen}

Este artículo tuvo como objetivo identificar y analizar estudios sobre la percepción de comodidad/malestar durante el uso de pantalones vaqueros por mujeres de mediana edad, a partir de una revisión sistemática de la literatura (RSL). La búsqueda de evidencias se realizó en las bases de datos Scopus, Web of Science y Periódicos Capes. Se utilizó un instrumento de RSL adaptado. Se seleccionaron diez artículos que cumplieron con los criterios de inclusión y calidad. Se constató que existen lagunas en las investigaciones relacionadas con las mujeres de mediana edad, la relación de este público y el uso de pantalones vaqueros y su percepción de comodidad/malestar, respondiendo parcialmente a la pregunta de investigación. Se identificaron limitaciones y se sugirió una mayor investigación que aborde el tema, contribuyendo a a las investigaciones y el desarrollo de productos de mezclilla y mejorando la calidad de vida de las usuarias.

Palabras clave: Ergonomía; Agradabilidad; Envejecimiento; Jeanswear. 
Research, Society and Development, v. 10, n. 14, e115101421734, 2021

(CC BY 4.0) | ISSN 2525-3409 | DOI: http://dx.doi.org/10.33448/rsd-v10i14.21734

\section{Introdução}

A maioria dos países em desenvolvimento vem passando por uma transição demográfica, com um envelhecimento gradativo da população, fenômeno resultante, dentre outros fatores, do aumento da expectativa de vida do ser humano. O envelhecimento é um processo natural da vida, classificado pela Organização Mundial da Saúde (OMS) em diferentes estágios: meia-idade (45 a 59 anos); idoso (60 a 89 anos); e velhice extrema (90 anos e acima) (Matsumoto et al., 2018). Entretanto, essa classificação cronológica determina tão somente a passagem do tempo, uma vez que o envelhecimento é uma condição complexa, que abrange transformações físicas, biológicas, psicológicas e também sociais (Schneider \& Irigaray, 2008). De fato, as pessoas estão vivendo cada vez mais e está ocorrendo inclusive um cenário global de "feminização" do envelhecimento, com um crescimento maior no número de mulheres que de homens (ONU, 2020; Cepellos, 2021).

Para as mulheres, o envelhecimento não indica apenas uma transição corporal, mas, sendo a feminilidade uma construção cultural, além da proximidade da menopausa e o término da ovulação, isso determina, ainda que simbolicamente, a "morte da juventude" (Veiga, 2017). As alterações biopsicossociais observadas afetam a autoimagem feminina e os desejos e necessidades envolvem questões emocionais e ergonômicas, particularmente quanto aos produtos de moda destinados às mulheres de meia-idade (Neves \& Paschoarelli, 2016). O vestuário atua como uma segunda pele e deve exercer uma função protetora, promovendo a segurança e o conforto do usuário (Martins, 2019), mas várias são as dificuldades enfrentadas pelas mulheres de meia-idade para encontrar roupas que sejam atrativas, adequadas aos seus corpos, com bom caimento e confortáveis (Tyagi \& Goel, 2013; Neves \& Paschoarelli, 2016), e um item de vestuário que elucida bem esse fato é a calça jeans.

O jeans é um produto de moda muito popular, aparentemente democrático e, no caso do Brasil, referência mundial, já que o País é o quarto maior produtor e consumidor de tecido denim ${ }^{1}$ no mundo, contudo, apesar de seu impacto social e econômico, a calça jeans ainda recebe críticas relacionadas ao ajuste corporal e ao desconforto por parte dos usuários, especialmente do público feminino (Theisen, Moura \& Folle, 2015; Abit, 2021). Isso acontece porque muitas inovações no vestuário, inclusive no segmento jeanswear, são voltadas mais para o aspecto estético e não são levadas em consideração as especificidades ergonômicas (Theisen, Moura \& Folle, 2015), como também as transformações corporais vivenciadas pelas usuárias de meia-idade. Mencionar desconforto de um produto de moda exige enfrentar desafios de conceituação, já que a sua análise pode se dar sob diferentes perspectivas. $\mathrm{O}$ desconforto pode ser caracterizado como a inexistência de conforto (Lueder, 1983), e, neste caso, Iida (2005) afirma que tal análise “(...) depende da área de estudo em que é aplicado” (p. 248). Neste sentido, Paschoarelli (2003) indica que a percepção de uso de determinado produto pode ser avaliada conforme critérios negativos e positivos, o que vai ao encontro da proposta de Slater (1986) para estes conceitos, ou seja, conforto é um estado agradável em que há harmonia física, fisiológica e psicológica entre o ser humano e seu meio.

Para Van der Linden (2003), a percepção de conforto abrange dimensões objetivas e subjetivas, pois o "sentir-se confortável" indica uma circunstância resultante do equilíbrio entre os aspectos físicos e os emocionais. Logo, adequações ergonômicas são indispensáveis nos produtos do segmento jeanswear, pois aspectos relacionados ao conforto agregam diversos benefícios e evidenciam resultados adequados e satisfatórios às peças finais, suprindo assim as necessidades e os anseios dos usuários (Martins, 2019). Diante do exposto, observou-se uma lacuna sobre estudos relacionados à temática, que envolvam as mulheres de meia-idade, e sua percepção de conforto/desconforto acerca da calça jeans. O presente estudo teve como objetivo identificar e analisar estudos sobre a percepção de conforto/desconforto, no uso da calça jeans, por mulheres de meia-idade, a partir de uma revisão bibliográfica sistemática (RBS).

\footnotetext{
${ }^{1}$ Denim é o tecido utilizado para produzir peças jeans. É uma sarja de algodão, feita em construção diagonal, com fio cru no sentido transversal (trama) e fio tinto no sentido longitudinal (urdume), sendo tingido por um corante de pigmento azul denominado "índigo" (Gorini, 1999; Catoira, 2006).
} 
Research, Society and Development, v. 10, n. 14, e115101421734, 2021

(CC BY 4.0) | ISSN 2525-3409 | DOI: http://dx.doi.org/10.33448/rsd-v10i14.21734

\section{Materiais e Métodos}

Trata-se de pesquisa de natureza teórica, exploratória e bibliográfica, com abordagem mista, uma vez que apresenta a análise quantitativa e qualitativa dos dados obtidos a partir de uma RBS. De acordo com Sampaio e Mancini (2007), RBS é uma forma de investigação que utiliza métodos sistematizados de busca, análise crítica e síntese da literatura sobre um tema, que “nos permitem incorporar um espectro maior de resultados relevantes, ao invés de limitar as nossas conclusões à leitura de somente alguns artigos” (p. 84). A análise quantitativa incluiu o estudo bibliométrico, a partir do número de citações, área de estudo dos periódicos, país de origem dos principais autores e ano de publicação; e a análise qualitativa abrangeu uma matriz de síntese, contendo a amostra de cada artigo, objetivos do estudo, método utilizado, principais resultados e escores de avaliação qualitativa.

O presente trabalho acompanhou a proposta das autoras supracitadas (ibidem, 2007) e a RBS foi realizada em cinco fases, a saber: (1) Definição da questão de pesquisa; (2) Busca da evidência nas bases de dados; (3) Avaliação e seleção dos estudos, seguindo os critérios de inclusão e exclusão definidos; (4) Análise da qualidade metodológica dos estudos incluídos, considerando as possíveis fontes de erro ou viés (bias); e (5) Apresentação dos resultados, com a inclusão e discussão das informações presentes nos artigos selecionados.

A primeira fase do processo consistiu na elaboração de uma pergunta feita com base na estratégia PICO, acrônimo para “População", "Intervenção", “Contexto” e "Outcomes” (desfecho), que é usada para direcionar a execução das outras atividades do processo e para auxiliar na resolução da questão de pesquisa (ibidem; Santos, Pimenta \& Nobre, 2007). Assim, foi formulado o seguinte questionamento: “As mulheres de meia-idade, ao usarem calça jeans, percebem diferentes níveis de conforto?”.

A segunda fase, que compreendeu a busca por evidências, foi realizada em três bases de dados distintas, a Scopus, a Web of Science e o Periódicos Capes. Foram definidas as palavras-chave no idioma inglês, baseadas na questão apresentada, utilizando-se vocabulários controlados e reconhecidos mundialmente, confirmados no Descritores em Ciências da Saúde (DeCS), assim como vocabulários não controlados, com termos variantes e sinônimos. Para a formação das strings de busca, em virtude de algumas limitações, como, por exemplo, restrição na quantidade de caixas de pesquisa para inserção da string completa, isto é, com a presença de todas as palavras-chave da questão, foi preciso realizar adaptações, de acordo com cada base, utilizandose, inclusive, mais de uma expressão. A disposição das strings apresenta-se organizada na Tabela 1.

Tabela 1 - Disposição das strings de busca.

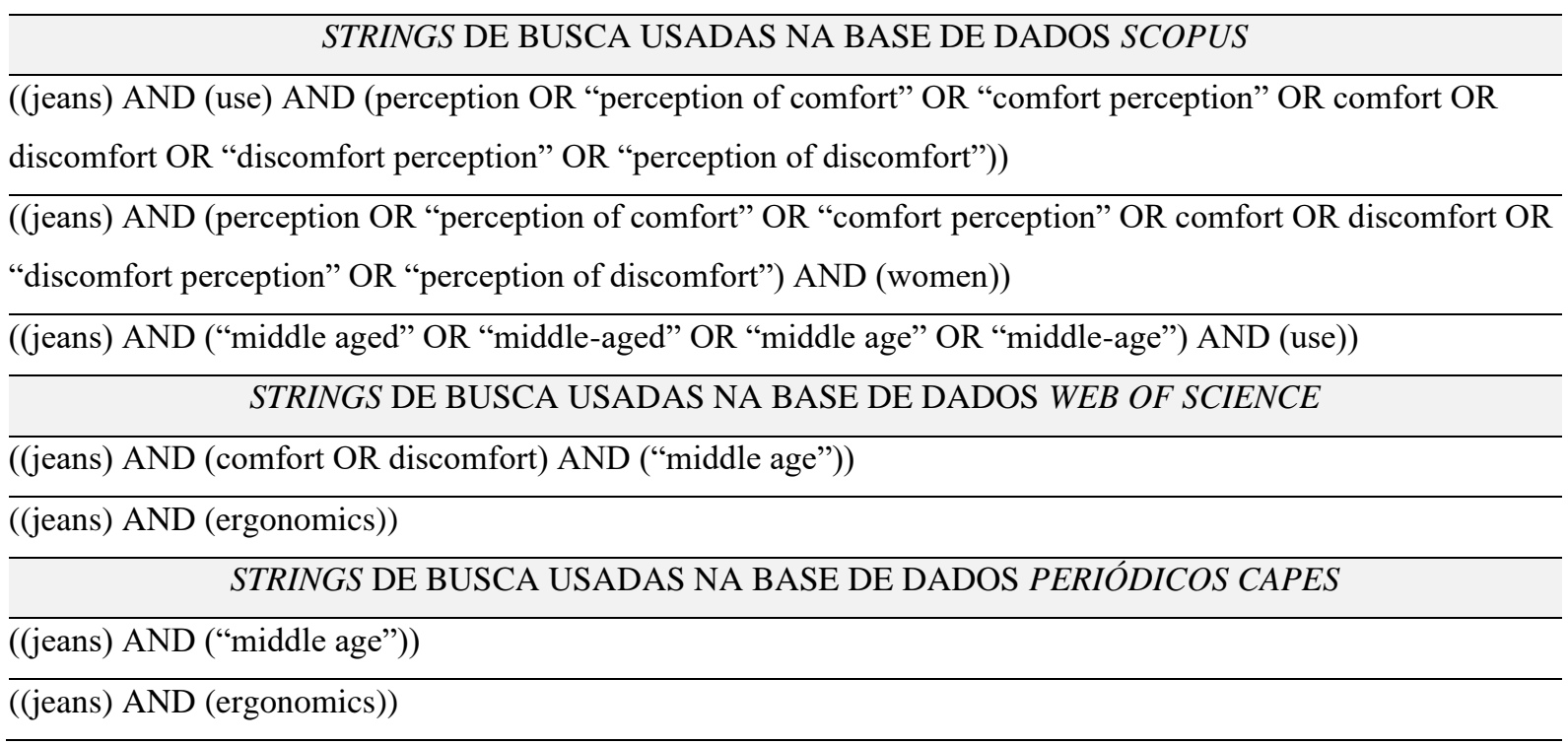

Fonte: Autores. 
Research, Society and Development, v. 10, n. 14, e115101421734, 2021

(CC BY 4.0) | ISSN 2525-3409 | DOI: http://dx.doi.org/10.33448/rsd-v10i14.21734

Na terceira fase, que diz respeito à avaliação e seleção dos estudos, foram definidos como critérios de inclusão: artigos científicos originais, publicados em periódicos revisados por pares e indexados às bases de dados, escritos nos idiomas português e inglês, entre as datas de publicação de 2005 a 2021, que fossem estudos sobre o conforto/desconforto do jeans ou relacionados à população definida (mulheres de meia-idade), e estudos que tratassem sobre alguma dimensão do conforto/desconforto associado ao uso do jeans. Logo, foram excluídos os artigos que não abrangessem os critérios propostos, ou que não tivessem relação com a temática.

Após a triagem inicial, apoiada nos critérios de inclusão e exclusão, verificou-se o título, resumo e palavras-chave dos resultados, dentro das próprias bases de dados. Com o remanescente, apurou-se a existência de artigos duplicados, para que, após essa nova supressão, fossem lidas a introdução e conclusão de cada estudo restante. Por fim, foram lidos na íntegra aqueles artigos elegíveis, e, então, selecionados dez artigos para serem avaliados criticamente, por meio de instrumento específico para tal finalidade, o Checklist Downs \& Black. Um esquema com o fluxograma dos resultados da busca, inclusão e seleção dos artigos presentes na RBS estão ilustrados na Figura 1.

Em seguida, passou-se para a quarta fase do processo, que analisou a qualidade e a validade desses estudos incluídos na RBS. De acordo com Pereira e Galvão (2014, p. 577), “A avaliação da qualidade dos estudos indicará a confiança que se terá nos resultados da revisão". Essa etapa consiste em uma avaliação do rigor metodológico, a fim de averiguar se os métodos e resultados de cada estudo são válidos e pertinentes para serem considerados.

Com isso, optou-se por utilizar os critérios de avaliação do instrumento denominado Checklist for measuring study quality, proposto por Downs e Black (1998), mas foram realizadas também adaptações, com 10 (dez) dos 27 itens originais, estruturados em forma de perguntas e agrupados em três categorias: "relato do estudo", que se refere à clareza dos trabalhos; "validade externa", acerca da representatividade; e "validade interna", referente ao viés de seleção. Cada resposta foi pontuada com o valor "1" (um), quando o resultado fosse positivo ("sim"), o que caracterizou a presença do critério de qualidade, ou "0" (zero), quando o resultado fosse negativo ("não" ou "incapaz de determinar"), o que caracterizou a ausência do critério de qualidade. Assim, o sistema de classificação adaptado compreendeu escores que foram de zero (pior qualidade) a dez (melhor qualidade). A Tabela 2 apresenta os itens usados para avaliação qualitativa dos artigos selecionados pelo Checklist Downs \& Black adaptada (Downs \& Black, 1998).

A quinta e última fase diz respeito à apresentação dos resultados, que serão expostos detalhadamente em seção própria deste artigo. Para organizar a extração dos dados, a fim de evitar retornos contínuos ao texto dos artigos selecionados, criou-se inicialmente uma planilha no dispositivo Microsoft Excel 2016® (Pereira \& Galvão, 2014), o que ajudou posteriormente na construção das duas matrizes de síntese, a da análise bibliométrica e a da análise do conteúdo. 
Tabela 2 - Checklist Downs \& Black adaptada (Downs \& Black, 1998).

\section{RELATO DO ESTUDO}

1. $\mathrm{O}(\mathrm{s})$ objetivo(s) do estudo foi claramente descrito?

2. Os principais resultados medidos foram claramente descritos na introdução ou na seção de métodos?

3. As características dos participantes incluídos no estudo foram claramente descritas?

4. Os principais resultados foram claramente descritos?

5. As características das perdas foram apresentadas?

\section{VALIDADE EXTERNA}

6. Houve representatividade na amostra do estudo?

7. Foi deixado claro no estudo algum resultado que não tenha sido baseado em hipótese planejada inicialmente?

8. Os principais métodos utilizados para mensurar os resultados foram válidos e confiáveis?

\section{VALIDADE INTERNA}

9. Os participantes recrutados pertenciam à mesma população?

10. Os participantes foram recrutados no mesmo período de tempo?

Fonte: Autores.

Figura 1 - Fluxograma da busca dos artigos.

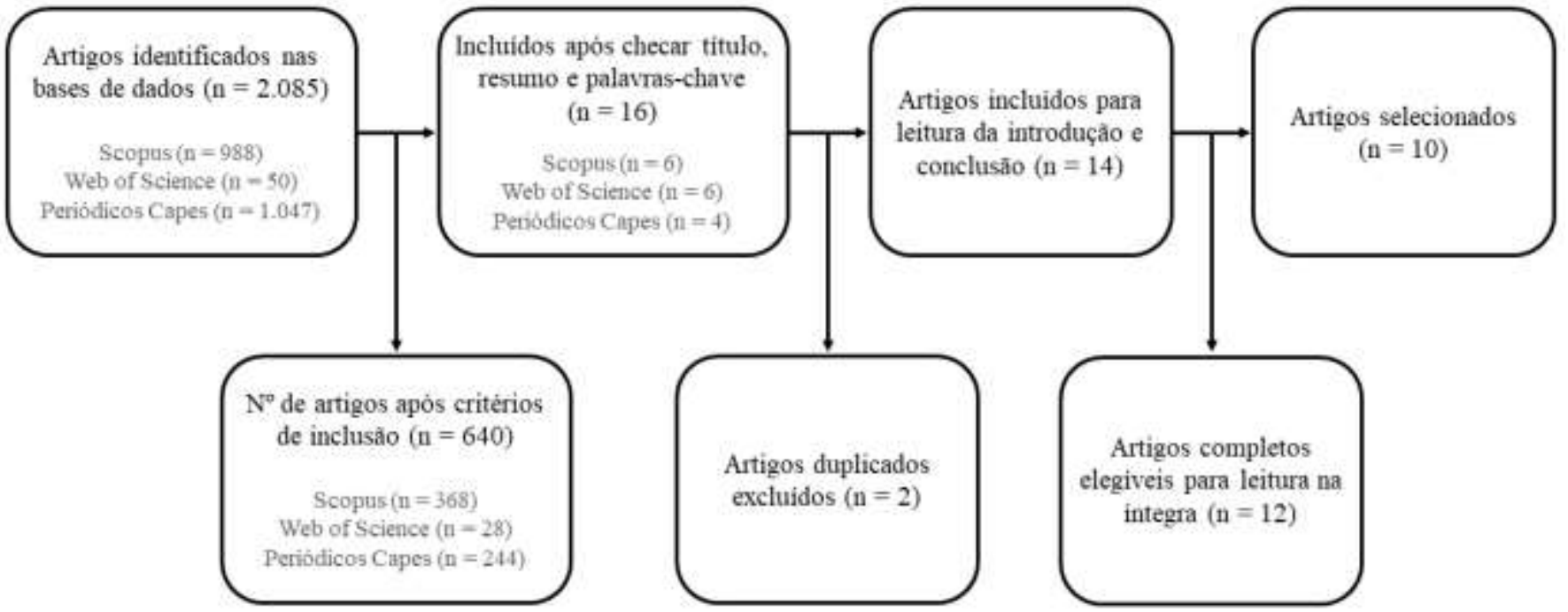

Fonte: Autores.

\section{Resultados}

Os resultados desta RBS foram divididos em quantitativos e qualitativos. No que se refere à parte quantitativa, apoiada por análise bibliométrica, a pesquisa foi realizada no dia 9 de junho do ano de 2021 nas bases de dados e retornou 2.085 artigos no total, sendo 988 da base Scopus, 50 da Web of Science e 1.047 do Periódicos Capes. Com base na aplicação dos critérios de inclusão, restaram 640 artigos (368 da Scopus; 28 da Web of Science; 244 do Periódicos Capes) e, após a checagem, em cada uma das bases de dados, do título, resumo e palavras-chave dos trabalhos restantes, resultaram16 artigos (6 da Scopus; 6 da Web of Science; 4 do Periódicos Capes). Foram excluídos 2 artigos duplicados e, com a leitura da introdução e conclusão desses 14 artigos, ficaram 12 estudos completos elegíveis para a leitura na íntegra. Entretanto, logo após essa leitura completa, dois dos 
artigos não correspondiam ao objetivo desta RBS e, assim, foram selecionados, ao final, 10 artigos, que atenderam aos critérios especificados anteriormente.

O número de citações por artigo foi verificado no Google Scholar (Google Acadêmico), conforme demonstra a Tabela 3, em 5 de julho de 2021, ordenado por número de citações em ordem decrescente. O artigo "Chinese perceptions of westernbranded denim jeans: a Shanghai case study” (Wu \& Delong, 2006) obteve 108 citações, sendo o mais citado até o momento da verificação, seguido pelo artigo “Comparison of chinese and indian consumers' evaluative criteria when selecting denim jeans: a conjoint analysis" (Jin, Park \& Ryu, 2010), com 85 citações, que foi o segundo mais citado. Todos os outros artigos receberam menos de 75 citações e apenas um artigo recebeu 5 citações, o "Understanding taiwanese female baby boomers through their perceptions of clothing and appearance" (Rahman \& Chang, 2018), representando o menor número desse grupo selecionado.

Com relação à área de estudo, a de Marketing foi a que obteve o maior número de ocorrências, com uma quantidade de cinco artigos. Uma publicação, que é multidisciplinar, envolveu abordagens nas áreas de Marketing e de Gestão e Moda. Foram selecionados também dois artigos pertencentes a periódicos específicos da área de Moda e dois da área de Design. Quanto à distribuição das publicações, levando-se em conta o país do primeiro autor: seis estudos do Canadá, três dos Estados Unidos e um da Turquia; e, no que diz respeito ao ano de publicação, três artigos foram no ano de 2010, dois em 2018 e, quanto ao restante, houve apenas um artigo publicado nos diferentes anos, feitos entre 2005 e 2021 . Além disso, quatro estudos foram desenvolvidos em colaboração com autores de diferentes países/instituições, de acordo com as informações detalhadas na Tabela 3 (Jin, Park \& Ryu, 2010; Howarton \& Lee, 2010; Rahman, Jiang \& Liu, 2010; Rahman, Fung, Chen \& Gao, 2017).

No que diz respeito à avaliação de qualidade dos trabalhos, destaca-se o artigo com maior pontuação - nove pontos (Howarton \& Lee, 2010), e os de menor pontuação - sete pontos (Wu \& Delong, 2006; Jin, Park \& Ryu, 2010; Rahman, Jiang \& Liu, 2010; Rahman, Fung, Chen \& Gao, 2017; Rahman \& Yu, 2018). De acordo com a apuração da Checklist Downs \& Black adaptada, a qualidade metodológica da maioria dos artigos revelou-se de média a boa. Na Tabela 4 são apresentados os resultados qualitativos, com as principais características dos dez estudos selecionados, incluindo os escores dessa avaliação de qualidade. A maior parte dos estudos (seis) eram surveys e, por esse motivo, foram utilizados questionários estruturados para a coleta de dados (Çoruh, 2009; Rahman, 2011; Howarton \& Lee, 2010; Rahman, Jiang \& Liu, 2010; Jin, Park \& Ryu, 2010; Rahman, Fung, Chen \& Gao, 2017). Dois estudos utilizaram métodos qualitativos, com entrevistas semiestruturadas (Rahman \& Chang, 2018; Rahman \& Yu, 2018); e outros dois com métodos combinados, sendo um de observação e survey (Wu \& Delong, 2006) e um que uniu entrevista e experimento, este por meio de estímulos visuais e táteis (Rahman, 2012).

Para as pesquisas quantitativas, a maior amostra foi a de Çoruh (2009), com um total de 1.170 participantes e a menor amostra foi a de Wu e Delong (2006), com 219 indivíduos participantes. Já para as pesquisas qualitativas, a pesquisa de Rahman (2012) foi realizada com 42 participantes, a de Rahman e Chang (2018) teve a participação de 14 indivíduos e a de Rahman e Yu (2018), um total de 13 pessoas, o que está em conformidade com o indicado por Cezar-Ferreira (2004, p. 88), quando aponta que "na pesquisa qualitativa, não há necessidade de grande número de participantes, uma vez que, nessa modalidade de pesquisa, a opção é pela profundidade em detrimento da amplitude". Ainda com relação às amostras, nem todas foram compostas por mulheres de meia-idade (45 a 59 anos). Na verdade, três estudos focaram na chamada "geração baby boomer" ${ }^{2}$, que engloba indivíduos que possuem a idade analisada nesta RBS (Howarton \& Lee, 2010; Rahman e Chang, 2018; Rahman \& Yu, 2018), e também o artigo de Wu e Delong (2006), que abrangeu participantes de 18 a 49 anos, abarcando parte desse grupo. Os outros estudos compreenderam jovens estudantes universitários, conforme descrito na Tabela 4, que foram incluídos nesta RBS porque

\footnotetext{
${ }^{2} \mathrm{O}$ sentido de geração atribuído nos estudos refere-se ao agrupamento de indivíduos que convive em uma mesma época, possui idades aproximadas e partilha algumas experiências de vida (Motta, 2010).
} 
atenderam outros critérios de inclusão, pois trataram sobre o conforto/desconforto do jeans ou sobre alguma dimensão do conforto associado ao jeans.

Tabela 3 - Artigos selecionados, ordenados pelo número de citações.

\begin{tabular}{|c|c|c|c|c|c|c|}
\hline $\begin{array}{l}\text { AUTORES } \\
\text { / ANO }\end{array}$ & $\begin{array}{l}\text { PAÍS DO } \\
1^{\circ} \text { AUTOR }\end{array}$ & $\begin{array}{l}\text { COPUBLICAÇÕES } \\
\text { (AUTORES DE } \\
\text { OUTROS PAÍSES/ } \\
\text { INSTITUIÇÕES) }\end{array}$ & TÍTULO & PERIÓDICO & $\begin{array}{l}\text { ÁREA DE } \\
\text { ESTUDO }\end{array}$ & CITAÇÕES \\
\hline $\begin{array}{l}\text { Wu \& } \\
\text { Delong } \\
(2006) \\
\end{array}$ & USA & 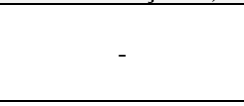 & $\begin{array}{c}\text { Chinese perceptions of } \\
\text { western-branded denim jeans: a } \\
\text { Shanghai case study } \\
\end{array}$ & $\begin{array}{c}\text { Journal of Fashion } \\
\text { Marketing and } \\
\text { Management } \\
\end{array}$ & Marketing & 108 \\
\hline $\begin{array}{l}\text { Jin, Park \& } \\
\text { Ryu (2010) }\end{array}$ & USA & $\begin{array}{l}\text { Estados Unidos; } \\
\text { Coréia do Sul }\end{array}$ & $\begin{array}{l}\text { Comparison of chinese and } \\
\text { indian consumers' evaluative } \\
\text { criteria when selecting denim } \\
\text { jeans: a conjoint analysis }\end{array}$ & $\begin{array}{l}\text { Journal of Fashion } \\
\text { Marketing and } \\
\text { Management }\end{array}$ & Marketing & 85 \\
\hline $\begin{array}{l}\text { Rahman } \\
(2012)\end{array}$ & USA & - & $\begin{array}{l}\text { The influence of visual and } \\
\text { tactile inputs on denim jeans } \\
\text { evaluation }\end{array}$ & $\begin{array}{c}\text { International Journal of } \\
\text { Design }\end{array}$ & Design & 73 \\
\hline $\begin{array}{l}\text { Rahman } \\
(2011)\end{array}$ & USA & - & $\begin{array}{l}\text { Understanding consumers' } \\
\text { perceptions and behaviors: } \\
\text { implications for denim jeans } \\
\text { design }\end{array}$ & $\begin{array}{c}\text { Journal of Textile and } \\
\text { Apparel, Technology and } \\
\text { Management }\end{array}$ & $\begin{array}{l}\text { Multidiscipli } \\
\text { nar (Moda, } \\
\text { Marketing, } \\
\text { Gestão) }\end{array}$ & 67 \\
\hline $\begin{array}{l}\text { Rahman, } \\
\text { Jiang \& Liu } \\
(2010) \\
\end{array}$ & Canadá & Canadá; China & $\begin{array}{l}\text { Evaluative criteria of denim } \\
\text { jeans: a cross-national study of } \\
\text { functional and aesthetic aspects }\end{array}$ & The Design Journal & Design & 51 \\
\hline $\begin{array}{l}\text { Rahman, } \\
\text { Fung, Chen } \\
\text { \& Gao } \\
(2017)\end{array}$ & Canadá & $\begin{array}{l}\text { Canadá; China; } \\
\text { Reino Unido }\end{array}$ & $\begin{array}{l}\text { A cross-national study of } \\
\text { apparel consumer preferences } \\
\text { and the role of product- } \\
\text { evaluative cues }\end{array}$ & $\begin{array}{l}\text { Asia Pacific Journal of } \\
\text { Marketing and Logistics }\end{array}$ & Marketing & 22 \\
\hline $\begin{array}{l}\text { Rahman \& } \\
\text { Yu (2018) }\end{array}$ & Canadá & - & $\begin{array}{l}\text { A study of canadian female } \\
\text { baby boomers: physiological } \\
\text { and psychological needs, } \\
\text { clothing choice and shopping } \\
\text { motives }\end{array}$ & $\begin{array}{l}\text { Journal of Fashion } \\
\text { Marketing and } \\
\text { Management }\end{array}$ & Marketing & 13 \\
\hline $\begin{array}{l}\text { Çoruh } \\
(2009)\end{array}$ & Turquia & - & $\begin{array}{l}\text { An investigation of the } \\
\text { ergonomics of jeans }\end{array}$ & $\begin{array}{c}\text { Tekstil ve Konfeksiyon } \\
\text { (Journal of Textile and } \\
\text { Apparel) } \\
\end{array}$ & Moda & 10 \\
\hline
\end{tabular}

Fonte: Autores.

Tabela 4 - Características dos estudos selecionados, incluindo os escores da avaliação de qualidade.

\begin{tabular}{|c|c|c|c|c|c|}
\hline $\begin{array}{l}\text { AUTOR/ } \\
\text { ANO }\end{array}$ & AMOSTRA & OBJETIVOS & MÉTODO & PRINCIPAIS RESULTADOS & $\begin{array}{c}\text { ESCORE } \\
\text { DOWNS \& } \\
\text { BLACK }\end{array}$ \\
\hline $\begin{array}{l}\text { Howarto } \\
\text { n \& Lee } \\
(2010)\end{array}$ & $\begin{array}{c}229 \text { mulheres } \\
\text { baby boomers } \\
\text { (nascidas entre } \\
1946-1964)\end{array}$ & $\begin{array}{l}\text { Investigar os } \\
\text { comportamentos de } \\
\text { compra e as atitudes das } \\
\text { baby boomers sobre suas } \\
\text { preferências do design das } \\
\text { roupas e os fatores que } \\
\text { motivam suas compras. } \\
\end{array}$ & $\begin{array}{c}\text { Survey } \\
\text { (questionário) }\end{array}$ & $\begin{array}{l}\text { - Falta atenção da indústria de vestuário às } \\
\text { necessidades das baby boomers quanto à } \\
\text { atratividade e ao ajuste das roupas; } \\
\text { - As baby boomers preferem roupas confortáveis } \\
(91,3 \%) \text {, com bom ajuste }(79,9 \%) \text { e lisonjeiras aos } \\
\text { seus corpos }(76,9 \%) \text {. }\end{array}$ & 9 \\
\hline $\begin{array}{l}\text { Çoruh } \\
(2009)\end{array}$ & $\begin{array}{l}1.170 \text { estudantes } \\
\text { universitários } \\
(614 \text { mulheres e } \\
556 \text { homens })\end{array}$ & $\begin{array}{c}\text { Investigar a ergonomia do } \\
\text { jeans. }\end{array}$ & $\begin{array}{c}\text { Survey } \\
\text { (questionário) }\end{array}$ & $\begin{array}{l}\text { - Foi constatado problema na área da cintura da } \\
\text { maioria dos jeans; } \\
\text { - Indicadas sugestões de modelagem para } \\
\text { solucionar problemas de desconforto (jeans deve } \\
\text { ser mais ergonômico, com melhor ajuste). }\end{array}$ & 8 \\
\hline $\begin{array}{l}\text { Rahman } \\
(2011)\end{array}$ & $\begin{array}{c}380 \text { mulheres } \\
\text { canadenses (18-24 } \\
\text { anos) }\end{array}$ & $\begin{array}{l}\text { Descobrir o significado dos } \\
\text { atributos intrínsecos e } \\
\text { extrínsecos como } \\
\text { determinantes da intenção } \\
\text { de compra dos }\end{array}$ & $\begin{array}{c}\text { Survey } \\
\text { (questionário) }\end{array}$ & $\begin{array}{l}\text { - O ajuste/caimento do jeans foi considerado o } \\
\text { atributo mais importante; } \\
\text { - O tecido foi fortemente relacionado ao estilo, } \\
\text { conforto e qualidade; }\end{array}$ & 8 \\
\hline
\end{tabular}


Research, Society and Development, v. 10, n. 14, e115101421734, 2021

(CC BY 4.0) | ISSN 2525-3409 | DOI: http://dx.doi.org/10.33448/rsd-v10i14.21734

\begin{tabular}{|c|c|c|c|c|c|}
\hline & & $\begin{array}{l}\text { consumidores em relação } \\
\text { aos jeans. }\end{array}$ & & $\begin{array}{l}\text { - Atributos intrínsecos (ex: conforto) } \\
\text { desempenharam um papel mais significativo na } \\
\text { avaliação de jeans do que os extrínsecos (ex: } \\
\text { marca). }\end{array}$ & \\
\hline $\begin{array}{l}\text { Rahman } \\
(2012)\end{array}$ & $\begin{array}{l}42 \text { mulheres } \\
\text { canadenses } \\
(18-27 \text { anos })\end{array}$ & $\begin{array}{l}\text { Investigar como os } \\
\text { recursos visuais e táteis } \\
\text { podem influenciar os } \\
\text { processos de avaliação dos } \\
\text { consumidores quando eles } \\
\text { compram um par de jeans. }\end{array}$ & $\begin{array}{l}\text { Métodos } \\
\text { combinados } \\
\text { (entrevista }+ \\
\text { experimento, } \\
\text { com } \\
\text { estímulos } \\
\text { visuais e } \\
\text { táteis) }\end{array}$ & $\begin{array}{l}\text { - Recursos, visuais e táteis influenciaram a } \\
\text { avaliação e o processo de tomada de decisão do } \\
\text { consumidor de jeans (recursos visuais ofereceram } \\
\text { aos consumidores uma impressão mais ampla e os } \\
\text { táteis puderam fortalecer e confirmar essas } \\
\text { percepções visuais); } \\
\text { - Consumidores usaram recursos funcionais } \\
\text { (concretos) para julgar a qualidade de um produto } \\
\text { e fizeram também uma conexão com valores } \\
\text { abstratos, como benefícios sociais, psicológicos e } \\
\text { sensoriais. }\end{array}$ & 8 \\
\hline $\begin{array}{l}\text { Rahman } \\
\text { \& Chang } \\
\text { (2018) }\end{array}$ & $\begin{array}{l}14 \text { mulheres baby } \\
\text { boomers } \\
\text { taiwaneses (idades } \\
\text { entre } 50 \text { e } 59 \\
\text { anos) }\end{array}$ & $\begin{array}{c}\text { Fornecer informações } \\
\text { sobre o impacto das } \\
\text { atitudes das baby boomers, } \\
\text { em Taiwan, quanto ao } \\
\text { envelhecimento, por meio } \\
\text { do vestuário, e entender } \\
\text { como as baby boomers em } \\
\text { Taiwan se comportam em } \\
\text { diferentes contextos quanto } \\
\text { à escolha, uso e consumo } \\
\text { de roupas. }\end{array}$ & $\begin{array}{l}\text { Entrevistas } \\
\text { semiestrutura } \\
\text { das. Após } \\
\text { entrevista, } \\
\text { cada } \\
\text { participante } \\
\text { foi convidada } \\
\text { a apresentar e } \\
\text { descrever seu } \\
\text { guarda-roupa. }\end{array}$ & $\begin{array}{l}\text { - Muitas baby boomers taiwaneses se } \\
\text { preocuparam com o pudor, a idade apropriada e o } \\
\text { conforto físico e psicológico quando se trata do } \\
\text { consumo de roupas; } \\
\text { - Conforto foi um dos fatores mais importantes } \\
\text { de avaliação de suas compras de roupas (facilidade } \\
\text { de movimento e propriedades do tecido da roupa } \\
\text { foram consideradas críticas). }\end{array}$ & 8 \\
\hline $\begin{array}{l}\text { Wu \& } \\
\text { Delong } \\
(2006)\end{array}$ & $\begin{array}{c}219 \text { participantes } \\
\text { (139 mulheres; } 79 \\
\text { homens) com } \\
\text { idades de } 18 \text { a } 49 \\
\text { anos }\end{array}$ & $\begin{array}{l}\text { Compreender as } \\
\text { percepções dos } \\
\text { consumidores e fornecer } \\
\text { informações gerenciais e } \\
\text { de marketing às empresas } \\
\text { ocidentais que vendem } \\
\text { jeans na China, } \\
\text { especificamente em } \\
\text { Xangai. }\end{array}$ & $\begin{array}{c}\text { Métodos } \\
\text { combinados } \\
\text { (observação + } \\
\text { survey) }\end{array}$ & $\begin{array}{l}\text { - Marcas de jeans ocidentais foram consideradas } \\
\text { confortáveis, com bons caimento e "design ou } \\
\text { corte"; } \\
\text { - Conforto }(31,51 \%) \text { foi o atributo dos jeans que } \\
\text { os consumidores chineses mais valorizavam, } \\
\text { seguido do ajuste }(22,37 \%) \text {. }\end{array}$ & 7 \\
\hline $\begin{array}{l}\text { Rahman, } \\
\text { Fung, } \\
\text { Chen \& } \\
\text { Gao } \\
(2017)\end{array}$ & $\begin{array}{l}440 \text { canadenses } \\
\text { (358 mulheres e } \\
75 \text { homens), com } \\
\text { idade média } 24,9 \\
\text { anos; } \\
484 \text { chineses ( } 282 \\
\text { mulheres e } 166 \\
\text { homens), com } \\
\text { idade média } 26,2 \\
\text { anos }\end{array}$ & $\begin{array}{l}\text { Identificar e investigar os } \\
\text { efeitos de saliência dos } \\
\text { atributos de avaliação do } \\
\text { vestuário, a fim de } \\
\text { enriquecer a compreensão } \\
\text { das preferências e do } \\
\text { comportamento do } \\
\text { consumidor em dois } \\
\text { contextos socioculturais } \\
\text { diferentes, Canadá e China. }\end{array}$ & $\begin{array}{l}\text { Survey } \\
\text { (questionário } \\
\text { online e em } \\
\text { papel) }\end{array}$ & $\begin{array}{l}\text { - Consumidores dos dois países estavam mais } \\
\text { preocupados com o ajuste e o estilo da roupa do } \\
\text { que com a marca e o país de origem; } \\
\text { - Para chineses, o conforto desempenhou um } \\
\text { papel mais significativo do que para os canadenses } \\
\text { (estes se preocupavam mais com a questão } \\
\text { estética); } \\
\text { - Ajuste, conforto e tecido foram fortemente } \\
\text { correlacionados, exceto no quesito "ajuste e } \\
\text { conforto" para os canadenses. }\end{array}$ & 7 \\
\hline $\begin{array}{l}\text { Rahman, } \\
\text { Jiang \& } \\
\text { Liu } \\
(2010)\end{array}$ & $\begin{array}{c}247 \text { mulheres } \\
\text { chinesas e } 380 \\
\text { mulheres } \\
\text { canadenses (18-24 } \\
\text { anos) }\end{array}$ & $\begin{array}{l}\text { Explorar e investigar os } \\
\text { atributos funcionais e } \\
\text { estéticos dos jeans e as } \\
\quad \text { percepções e } \\
\text { comportamentos gerados } \\
\text { no mercado jovem. }\end{array}$ & $\begin{array}{c}\text { Survey } \\
\text { (questionário) }\end{array}$ & $\begin{array}{l}\text { - Chinesas estavam mais preocupadas com as } \\
\text { características funcionais dos jeans, que } \\
\text { oferecessem status social aceitável, boa qualidade } \\
\text { e valor funcional; } \\
\text { - Canadenses focaram mais fortemente no apelo } \\
\text { estético e uso de roupas para construir sua } \\
\text { identidade, expressar o self e melhorar a aparência } \\
\text { externa, com foco maior nas necessidades pessoais. }\end{array}$ & 7 \\
\hline $\begin{array}{l}\text { Rahman } \\
\& \text { Yu } \\
(2018)\end{array}$ & $\begin{array}{l}13 \text { mulheres baby } \\
\text { boomers } \\
\text { canadenses } \\
\text { (idades entre } 53 \mathrm{e} \\
71 \text { anos) }\end{array}$ & $\begin{array}{c}\text { Compreender as } \\
\text { necessidades fisiológicas e } \\
\text { psicológicas das baby } \\
\text { boomers por meio do } \\
\text { consumo de roupas. }\end{array}$ & $\begin{array}{l}\text { Entrevistas } \\
\text { semiestrutura } \\
\quad \text { das }\end{array}$ & $\begin{array}{l}\text { Estilo, ajuste, conforto e cor foram os atributos } \\
\text { de avaliação de produto mais importantes; } \\
\text { - Designers de moda devem atender a idade } \\
\text { cognitiva das baby boomers, pois isso pode } \\
\text { aumentar a aceitação, autoconfiança e a satisfação } \\
\text { das consumidoras. }\end{array}$ & 7 \\
\hline $\begin{array}{l}\text { Jin, Park } \\
\text { \& Ryu } \\
(2010)\end{array}$ & $\begin{array}{l}302 \text { estudantes } \\
\text { universitários } \\
(152 \text { da China, } \\
\text { com uma media } \\
\text { de idade de } 20,8 \\
\text { anos; e } 150 \text { da } \\
\text { Índia, com uma } \\
\text { media de idade de } \\
22,1 \text { anos }) \\
\end{array}$ & $\begin{array}{l}\text { Comparar os atributos de } \\
\text { avaliação que os } \\
\text { consumidores chineses e } \\
\text { indianos utilizam ao } \\
\text { comprar jeans. }\end{array}$ & $\begin{array}{c}\text { Survey } \\
\text { (questionário) }\end{array}$ & $\begin{array}{l}\text { - Consumidores chineses atribuíram maior } \\
\text { importância ao preço, seguido por ajuste, país de } \\
\text { origem, qualidade e design, enquanto que a ordem } \\
\text { para os indianos foi de ajuste, país de origem, } \\
\text { design, preço e qualidade. } \\
\text { - Os resultados indicaram que esses dois grupos } \\
\text { de consumidores atribuíam importâncias distintas } \\
\text { aos atributos de avaliação do vestuário, e, portanto, } \\
\text { precisam ser tratados de forma diferente. }\end{array}$ & 7 \\
\hline
\end{tabular}

Fonte: Autores. 


\section{Discussão}

O presente estudo buscou, por meio de uma RBS, identificar e analisar estudos que realizaram abordagens sobre a percepção de conforto/desconforto do uso de calça jeans, por mulheres de meia-idade. Após aplicação de procedimento metodológico próprio de RBS, resultaram dez trabalhos de destaque nesta área.

Apenas um dos estudos selecionados é voltado especificamente para a investigação do aspecto ergonômico do jeans, em que foi constatado problema na área da cintura, na realização das atividades diárias dos usuários, especialmente na calça jeans feminina (Çoruh, 2009). Embora tenha sido uma pesquisa realizada com jovens universitários, o estudo reconhece e aponta outros trabalhos realizados sobre a insatisfação com o ajuste de roupas expressa também por mulheres idosas. Como solução, determinou algumas sugestões para uma nova modelagem do jeans, mais ergonômica, a fim de solucionar os problemas encontrados durante a realização da pesquisa.

De acordo com a autora, os problemas relacionados ao desconforto devem ser solucionados para melhorar a qualidade das roupas e para que as expectativas dos consumidores sejam atendidas, até mesmo pelo fato de que, independentemente das tendências e variações da moda, o jeans é um item de vestuário que continua sendo muito usado por todas as pessoas. As descobertas do estudo de Rahman (2011) revelaram justamente que mais da metade de todas as entrevistadas usava jeans diariamente ou quase todos os dias, além de possuírem de nove a dez calças jeans.

No entanto, chama a atenção que a maior parte dos estudos apresentados nesta RBS tiveram como amostra indivíduos mais jovens, o que evidencia uma lacuna de pesquisas feitas com o público de meia-idade, assim como relacionadas ao segmento jeanswear. Além disso, conforme o estudo de Howarton e Lee (2010), foi indicado que a indústria do vestuário negligencia esse público de mais idade, quanto à atratividade e ao ajuste de roupas. As participantes, que pertenciam à geração baby boomer, revelaram a preferência por roupas confortáveis $(91,3 \%)$, com bom ajuste $(79,9 \%)$ e lisonjeiras aos seus corpos $(76,9 \%)$.

A preferência pelo conforto foi corroborada por pesquisa anterior, de Wu e Delong (2006), em que este atributo foi o mais lembrado (31,51\%), seguido pelo ajuste (22,37\%), em resposta à pergunta aberta solicitando que o participante descrevesse seus sentimentos quando vestia um jeans de marca ocidental. Em estudo posterior, de Rahman e Yu (2018), as opções foram confirmadas novamente, indicando que, além do conforto, ajuste, estilo e cor foram os atributos de avaliação de produto mais importantes para as entrevistadas, estas também do grupo geracional baby boomer. Foi deixada uma recomendação para os designers de moda, sobre a importância de criar produtos de vestuário com base na idade cognitiva, pois isso influencia também no conforto psicológico e pode aumentar a aceitação, autoconfiança e satisfação dessas consumidoras baby boomers, o que leva a uma melhor qualidade de vida.

Em se tratando do jeans, de acordo com Rahman (2011, p.11), "os designers de moda devem prestar atenção a esses diversos valores dimensionais, em vez de meramente os aspectos concretos ou físicos de um produto". Para este autor, os indivíduos se preocupam com a sua imagem pessoal e o jeans foi usado como um item para realçar certas partes do corpo ou para esconder suas falhas, de maneira que se sentissem bem com sua aparência e imagem externas. É importante notar, dessa forma, que os consumidores/usuários estão constantemente em busca do conforto em sua multidimensionalidade, englobando também o aspecto emocional (conforto psicológico).

Rahman (2012) indicou que os recursos visuais e táteis influenciam na avaliação e no processo de tomada de decisão do consumidor de jeans, de maneira que o tátil fortalece e confirma o que o visual sugeriu. De acordo com as descobertas, os recursos táteis foram associados ao conforto, à durabilidade e a sensações agradáveis, e essa experiência sensorial positiva pode aumentar a resposta afetiva dos usuários. Além da conexão com valores abstratos, como benefícios sociais, psicológicos e sensoriais, certamente os consumidores usam os próprios recursos funcionais (concretos) para julgar a qualidade de um produto, 
o que também foi indicado pelos participantes, quando afirmaram que a quantidade de elasticidade (stretch) em uma calça jeans estava ligada ao seu ajuste, à retenção da forma e ao conforto proporcionado.

Mesmo em um contexto específico, como o da pesquisa de Wu e Delong (2006), que explorou o cenário chinês, com consumidores de Xangai, foi demonstrado que o conforto é o atributo mais valorizado em um jeans, seguido pelo seu ajuste. De acordo com as autoras, o jeans, por ser uma das categorias de roupas mais populares no mundo, é um item que contribui bastante na compreensão das percepções dos consumidores em uma cultura específica. Nesse sentido, outros trabalhos que também exploraram culturas distintas e suas relações com o jeans, como o de Rahman e Chang (2018), sobre as consumidoras chinesas baby boomers, de Taiwan, relataram que o conforto era um dos fatores mais importantes de avaliação de suas compras de roupas, principalmente no que se refere à facilidade de movimento e às propriedades do tecido das roupas.

Em outro estudo, Rahman (2011) indicou que o ajuste/caimento do jeans foi considerado o atributo mais importante e o tecido foi fortemente relacionado ao estilo, à qualidade e ao conforto, sendo este considerado um atributo intrínseco que desempenha um papel significativo na avaliação do jeans, mais até do que atributos extrínsecos, como, por exemplo, a marca do jeans.

O ajuste também foi classificado como o atributo mais importante para os consumidores indianos e o segundo mais importante para os consumidores chineses na pesquisa de Jin, Park e Ryu (2010), da mesma forma que na de Rahman, Fung, Chen e Gao (2017), que investigou o comportamento dos consumidores do Canadá e China, os resultados indicaram que os consumidores dos dois países estavam mais preocupados com o ajuste e o estilo da roupa do que outros atributos, sendo que para os chineses, o conforto desempenhou um papel relativamente mais significativo do que para os canadenses.

Para Rahman, Jiang e Liu (2010), que também haviam pesquisado consumidoras chinesas e canadenses, os resultados indicaram que as chinesas estavam mais preocupadas com as características funcionais dos jeans, como facilidade de movimento e conforto, aumentando o valor percebido do produto; já as canadenses estavam mais preocupadas com seu eu individual, pois os valores estéticos desempenhavam um papel significativo na avaliação e consumo de jeans, baseada em benefícios psicológicos. De acordo com os autores, “se o jeans não satisfizer os requisitos de imagem do consumidor, ele também não será percebido como bem cortado ou confortável” (p. 303).

\section{Considerações Finais}

O presente artigo se propôs a identificar e analisar estudos sobre a percepção de conforto/desconforto, no uso da calça jeans, por mulheres de meia-idade, a partir de uma RBS. Constatou-se que os pressupostos de lacunas acerca de pesquisas ligadas especificamente às mulheres de meia-idade foram confirmados, assim como a relação desse público com o uso da calça jeans e sua percepção de conforto/desconforto, o que permitiu responder parcialmente à questão de pesquisa.

Pelo fato de que as mulheres de meia-idade passam por várias transformações biopsicossociais durante o processo de envelhecimento, é preciso uma adequação técnica a essas novas condições, assim como a busca pela satisfação dos anseios psicológicos e sociais, proporcionando o conforto pleno a essas usuárias. Contudo, a maior parte dos estudos selecionados são ligados à área de marketing, os quais verificam os desejos dos consumidores muitas vezes pela percepção de compra, mais do que propriamente o lado do uso, sem levar tanto em consideração a usabilidade da peça e seu consequente conforto/desconforto. Notou-se que as pesquisas não consideraram todas as dimensões do conforto, por isso, há carência de artigos relacionados ao aspecto fisiológico, envolvendo a análise do conforto/desconforto térmico, por exemplo. Além disso, é importante mencionar que, apesar da notoriedade do segmento jeanswear brasileiro, não foi encontrado nenhum artigo de base nacional.

Foram identificadas limitações, como a restrição nos anos de busca, que, embora tenha sido um espaço de tempo relativamente considerável, estudos relevantes, anteriormente publicados ao período averiguado, podem ter sido ignorados, uma 
Research, Society and Development, v. 10, n. 14, e115101421734, 2021

(CC BY 4.0) | ISSN 2525-3409 | DOI: http://dx.doi.org/10.33448/rsd-v10i14.21734

vez que o jeans foi e continua a ser usado por diversas pessoas em praticamente todo o mundo, desde a sua criação. Nesse sentido, recomenda-se, para futuros trabalhos, o aumento no intervalo de tempo, o acréscimo de diferentes bases de dados científicos, a inclusão de outros idiomas, a inserção da literatura "cinzenta", como teses e dissertações, e ainda a utilização de termos distintos e sinônimos para a formação de novas strings; tudo isso irá provavelmente retornar resultados diversos da seleção incluída na presente RBS e irá promover o desenvolvimento e o aprimoramento de mais estudos que contemplem a temática.

Espera-se que esta revisão possa contribuir na orientação de designers, ergonomistas e demais pesquisadores interessados nas áreas para o crescimento das pesquisas, avanço no desenvolvimento de produtos de vestuário, em especial do jeans, e a consequente melhoria no bem-estar e na qualidade de vida de todos os usuários.

\section{Agradecimentos}

O presente estudo foi desenvolvido com apoio do CNPq - Conselho Nacional de Desenvolvimento Científico e Tecnológico - Processo 304619/2018-3.

\section{Referências}

Associação Brasileira da Indústria Têxtil e de Confecção - Abit (2021). Perfil do Setor - Dados gerais do setor referentes a 2019 (atualizados em dezembro de 2020). http://www.abit.org.br/cont/perfil-do-setor .x

Catoira, L. (2006). Jeans, a roupa que transcende a moda. Ideias \& Letras.

Cepellos, V. M. (2021). Feminização do envelhecimento: um fenômeno multifacetado muito além dos números. Revista de Administração de Empresas, 61(2). https://doi.org/10.1590/s0034-759020210208

Cezar-Ferreira, V. A. M. (2004). A pesquisa qualitativa como meio de produção de conhecimento em psicologia clínica, quanto a problemas que atingem a família. Psicologia: teoria e prática, 6(1), 81-95. http://pepsic.bvsalud.org/scielo.php?script=sci_arttext\&pid=S1516-36872004000100007\&lng=pt\&tlng=pt.

Çoruh, E. (2009). An investigation of the ergonomics of jeans. Textile and Apparel, 19 (3), 248-254. https://dergipark.org.tr/en/pub/tekstilvekonfeksiyon/issue/23635/251743.

Descritores em Ciências da Saúde - DeCS (2017). ed. São Paulo (SP): BIREME / PAHO / WHO. 2017. Acesso em: 9 jun. 2021. https://decs.bvsalud.org/.

Downs, S. H., \& Black, N. (1998). The feasibility of creating a checklist for the assessment of the methodological quality both of randomised and non-randomised studies of health care interventions. Journal of Epidemiology \& Community Health, 52(6), 377-384. http://dx.doi.org/10.1136/jech.52.6.377.

Gorini, A. P. F. (1999). O segmento de índigo. BNDES Setorial, (10), 313-334. http://web.bndes.gov.br/bib/jspui/handle/1408/3161.

Rahman, O. \& Yu, H. (2018). A study of Canadian female baby boomers: Physiological and psychological needs, clothing choice and shopping motives. Journal of Fashion Marketing and Management: An International Journal, 22(4), 509-526. https://doi.org/10.1108/JFMM-09-2017-0100.

Iida, I. (2005). Ergonomia: projeto e produção (2a ed.). Edgar Blücher.

Jin, B., Park, J. Y., \& Ryu, J.S. (2010). Comparison of Chinese and Indian consumers' evaluative criteria when selecting denim jeans: A conjoint analysis. Journal of Fashion Marketing and Management: An International Journal, 14(1), 180-194. https://doi.org/10.1108/13612021011025492.

Lueder, R. K. (1983). Seat Comfort: A Review of the Construct in the Office Environment. Human Factors: The Journal of the Human Factors and Ergonomics Society, 25(6), 701-711. https://doi.org/10.1177/001872088302500607.

MARTINS, S. B. (2019). Ergonomia, usabilidade e conforto em projeto de produto de moda e vestuário. In S. B. MARTINS (Org.), Ergonomia, usabilidade e conforto no design de moda: a metodologia OIKOS (pp. 56-77). Estação das Letras e Cores.

Matsumoto, W. K., Munhoz, A. M., Okada, A., Montag, E., Arruda, E. G., Fonseca, A., Ferrari, O., Brasil, J. A., Pretti, L., Filassi, J. R., \& Gemperli, R. (2018). Influence of advanced age on postoperative outcomes and total loss following breast reconstruction: a critical assessment of 560 cases. Revista do Colegio Brasileiro de Cirurgioes, 45(2), e1616. https://doi.org/10.1590/0100-6991e-20181616.

Motta, A. B. (2010). A atualidade do conceito de gerações na pesquisa sobre o envelhecimento. Sociedade e Estado, 25(2), 225-250. https://doi.org/10.1590/S0102-69922010000200005

Neves, E. P. \& Paschoarelli, L. C. (2016). Moda e meia idade: percepções femininas sobre o produto do vestuário da atualidade. Modapalavra E-periódico, 9(18), 192-205. https://doi.org/10.5965/1982615x09182016192.

Organização das Nações Unidas - ONU (2020). World Population Ageing 2020 Highlights: Living arrangements of older persons (ST/ESA/SER.A/451). United Nations Department of Economic and Social Affairs 2020. https://www.un.org/development/desa/pd/news/world-population-ageing-2020-highlights. 
Research, Society and Development, v. 10, n. 14, e115101421734, 2021

(CC BY 4.0) | ISSN 2525-3409 | DOI: http://dx.doi.org/10.33448/rsd-v10i14.21734

Paschoarelli, L. C. (2003). Usabilidade aplicada ao design ergonômico de transdutores de ultrassonografia: uma proposta metodológica para avaliação e análise do produto. [Tese (Doutorado), Universidade Federal de São Carlos]. Repositório Institucional UFSCar. https://repositorio.ufscar.br/handle/ufscar/3311

Pereira, M. G., \& Galvão, T. F. (2014). Extração, avaliação da qualidade e síntese dos dados para revisão sistemática. Epidemiologia e Serviços de Saúde, 23(3), 577-578. http://scielo.iec.gov.br/scielo.php?script=sci_arttext\&pid=S1679-49742014000300021\&lng=pt\&tlng=pt.

Rahman, O., Jiang, Y. \& Liu, W.-S. (2010). Evaluative Criteria of Denim Jeans: A Cross-national Study of Functional and Aesthetic Aspects, The Design Journal, 13(3), 291-311. https://doi.org/10.2752/146069210X12766130824894.

Rahman, O. (2011). Understanding consumers' perceptions and behaviors: implications for denim jeans design. Journal of Textile and Apparel, Technology and Management, 7(1), 1-16. https://ojs.cnr.ncsu.edu/index.php/JTATM/issue/view/38.

Rahman, O. (2012). The influence of visual and tactile inputs on denim jeans evaluation. International Journal of Design, 6(1), 11-25. http://www.ijdesign.org/index.php/IJDesign/issue/view/30.

Rahman, O., Fung, B. C. M., Chen, Z. \& Gao, X. (2017). A cross-national study of apparel consumer preferences and the role of product-evaluative cues. Asia Pacific Journal of Marketing and Logistics, 29(4), 796-812. https://doi.org/10.1108/APJML-09-2016-0175.

Rahman, O., \& Chang, W.-T. (2016). Understanding Taiwanese Female Baby Boomers through their Perceptions of Clothing and Appearance. Fashion Practice, 10(1), 53-77. https://doi.org/10.1080/17569370.2016.1190102.

Rahman, O., \& Yu, H. (2018). A study of Canadian female baby boomers. Journal of Fashion Marketing and Management: An International Journal, 22(4), 509-526. https://doi.org/10.1108/jfmm-09-2017-0100.

Sampaio, R. F. \& Mancini, M. C. (2007). Estudos de revisão sistemática: um guia para síntese criteriosa da evidência científica. Revista Brasileira de Fisioterapia, 11(1), 83-89. https://doi.org/10.1590/S1413-35552007000100013.

Santos, C. M. C., Pimenta, C. A. M. \& Nobre, M. R. C. (2007). A estratégia PICO para a construção da pergunta de pesquisa e busca de evidências. Revista Latino-Americana de Enfermagem, 15(3), 508-511. https://doi.org/10.1590/S0104-11692007000300023.

Schneider, R. H. \& Irigaray, T. Q. (2008). O envelhecimento na atualidade: aspectos cronológicos, biológicos, psicológicos e sociais. Estudos de Psicologia (Campinas), 25(4), 585-593. https://doi.org/10.1590/S0103-166X2008000400013.

Slater, K. (1986). Discussion Paper - The assessment of comfort. The Journal of The Textile Institute, 77(3), 157-171. https://doi.org/10.1080/00405008608658406.

Theisen, F. C., Moura, H. T. \& Folle, L. F. (2015). Inovação no design de moda: articulando antropometria, ergonomia e usabilidade para conforto no uso da calça jeans. Strategic Design Research Journal, 8(3), 116-126. https://doi.org/10.4013/sdrj.2015.83.02.

Tyagi, I. \& Goel, A. (2013). Factors affecting clothing choices of elderly females. Indian Journal of Gerontology, 27(2,) 307-319. http://www.gerontologyindia.com/pdf/vol27-3.pdf.

Van der Linden, J. C. (2003). O conceito de conforto. Revista Tecnologia e Tendências, 2(2), 21-30. https://doi.org/10.25112/rtt.v2i2.

Veiga, M. R. M. (2017). Envelhecer no feminino: corpo e feminilidade na maturidade. In L. S. Ferreira, \& Z. N. Borges (Orgs.), Mosaico socioantropológico: temas transversais de cultura, gênero e saúde (Vol. 1, pp. 84-92). Editora Experimental pE.com.

Wu, J. \& Delong, M. (2006). Chinese perceptions of western-branded denim jeans: a Shanghai case study. Journal of Fashion Marketing and Management: An International Journal, 10(2), 238-250. https://doi.org/10.1108/13612020610667531. 